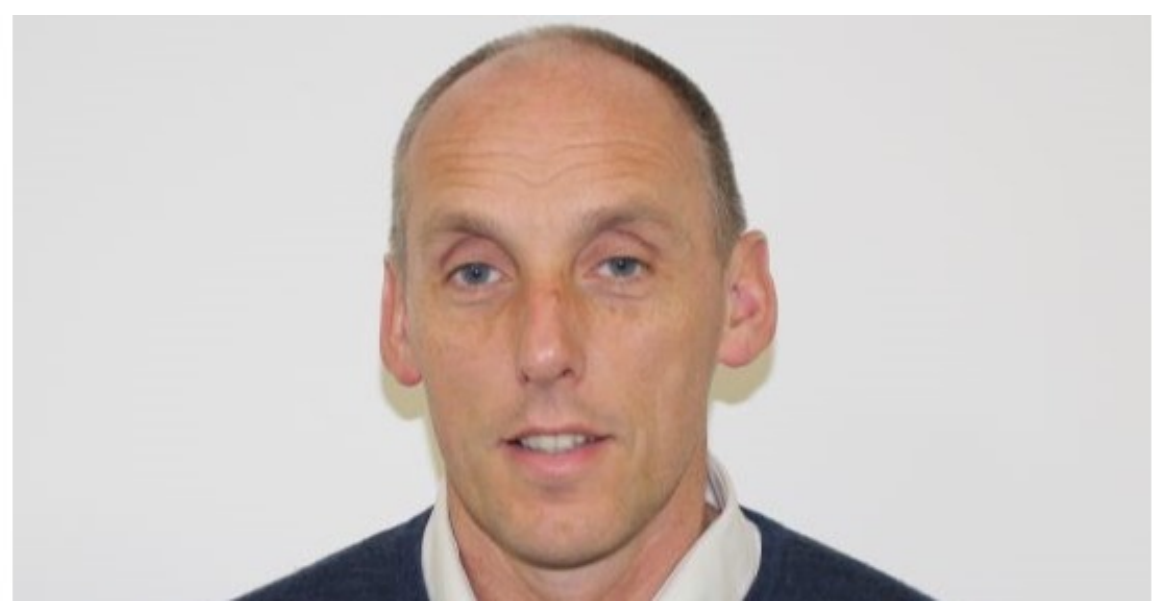

\title{
Long-term Condition Management for Prisoners: exploring prevalence and compliance with national monitoring processes
}

Nat Wright, Victoria Allgar, Frances Hankins, Philippa Hearty

Funding: The work was funded by NHS England Health and Justice

Potential competing interests: The author(s) declared that no potential competing interests exist.

\section{Abstract}

Abstract

Background: Long-term conditions (LTCs) are a significant cause of morbidity and mortality and prisoner populations have a disproportionately high prevalence of risk factors for LTCs. The size and mean age of the prison population has increased rapidly in recent years. The UK Quality Outcomes Framework (QOF) is a national standardised framework embedded in community general practice with financial remuneration linked to assessment and ongoing review of key clinical outcomes pertaining to LTCs. However, healthcare in prisons in England is not linked to financial remuneration through the QOF framework and prison clinicians are not mandated to adhere to the framework.

Aim: To explore prevalence of LTCs in remand prisons and measure compliance with QOF monitoring.

Design and Setting: Quantitative analysis of secondary data on SystmOne.

Methods: Secondary data analysis of data extracted from the prison primary care record pertaining to patient self-report of LTC, level of confirmation by supporting evidence and compliance with QOF monitoring frameworks.

Results: $17 \%$ of the sample had at least one LTC, the most common condition being asthma, confirmed in $12 \%$ of the sample. Having an LTC was associated with female gender and increasing age. Prevalence rates for the other LTCs were hypertension $3 \%$, epilepsy was $3 \%$, coronary heart disease $2 \%$, diabetes $2 \%$ and chronic obstructive pulmonary 
disease $1 \%$. Just $34 \%$ of the eligible sample had had a QOF template completed. Higher rates of completion were associated with younger age and there were also statistically significant inter-prison differences.

Conclusion: There is a pressing need to embed standardised QOF monitoring systems within an integrated community/prison commissioning framework supported by connectivity between prison and community primary care records of not just the summary care record but also all activity related to QOF compliance.

\section{Introduction}

Long-term conditions (LTCs) are those that cannot be cured but are controlled through medication and/or other forms of therapy. ${ }^{[1]}$ It is estimated that more than 15 million people in the UK suffer from a long-term condition, ${ }^{[2]}$ with multimorbidity also becoming increasingly problematic. ${ }^{[1]}$

The risk factors for such LTCs disproportionately affects prisoner populations. ${ }^{[3]}$ Currently in England and Wales there are over 83,000 individuals imprisoned. ${ }^{[4]}$ Compared to equivalent community populations, prisoners consult primary care doctors three times more frequently, consult other primary health care workers 80 times more frequently, and receive inpatient care at least 10 times more frequently. ${ }^{[5]}$ They have a higher mortality and morbidity rates from chronic disease. ${ }^{[6]}$

Internationally, ethical codes of practice highlight a "principle of equivalence" which states that prisoners have a right to an equivalent quality of healthcare as they would receive in the community. ${ }^{[7]}$ However, in practice there are significant threats to providing such equivalent healthcare. For example, medical indemnity organisations acknowledge that patients in prison may be examined and treated in situations that are far from the norm for the rest of society. They provide examples of consultations which may take place without access to GP or hospital records or may be held in an environment that could compromise safety for both patient and doctor. ${ }^{[8]}$ This is particularly commonplace when patients enter remand prisons outside of normal working hours and are assessed in first night prison reception centres. In such an environment there are significant threats to effective medicines management - defined as "a system of processes and behaviours that determines how medicines are used by patients and by the NHS". ${ }^{[9]}$ Prisoners commonly enter prison without their medication prescribed for their LTC and confirming their medication with community GP services is not possible outside of normal working hours. Further, once medication is confirmed and prescribed there are potential delays in both time to first dispense and time to first administration of such medication. With the exception of opiate substitution treatment, there are no national guidelines to inform best practice in terms of when it is appropriate to offer substitute medications where medication cannot be confirmed on first night reception areas. By implication, there are no guidelines to inform clinicians when it is appropriate to withhold medication pending confirmation by a third party.

Regarding assessment for those entering prison, there is a process of healthcare screening through both "first" and "second" tools that are integral to the patient record. The former, addresses issues pertaining to acute health need (e.g. demographic details; assessment of withdrawal from drugs or alcohol; contact details for community pharmacists to enable 
timely confirmation of outstanding prescriptions), whereas the second screen covers an assessment of the need for ongoing management of long-term conditions and current immunisation status.

Once patients have undergone assessment and are established in prison there is an opportunity to obtain supporting evidence to confirm their self-report of an LTC by obtaining confirmation from either their community GPs or arranging the necessary clinical tests, thus permitting the health risks posed by LTCs to be more proactively managed. In community general practice in England, the key framework to achieve this objective is the Quality and Outcomes Framework ${ }^{[10]}$ (in Scotland such information is collected and presented through Primary Care Information Dashboards ${ }^{[11]}$ ). Such a national standardised framework is now embedded in community general practice with financial remuneration linked to assessment and ongoing review of key clinical outcomes pertaining to LTCs. However, healthcare in prisons in England is not linked to financial remuneration through the QOF framework and prison clinicians are not mandated to adhere to the framework. Rather, the only mandated process is that of all prisoners upon reception into prison undergoing the screening process outlined above. Such a screening process places less of an emphasis upon clinical outcomes than that outlined in the QOF framework. Therefore, since compliance with QOF monitoring processes is voluntary in prisons, it is possible that an opportunity to improve clinical outcomes associated with LTCs is being missed. Therefore, in response to such a gap in service provision, we explored the topic of the assessment and management of LTCs in four remand prisons. By extracting routinely collected clinical data, we explored the prevalence of LTCs, compliance with both first and second assessment, and also QOF monitoring processes.

\section{Methods}

After acquiring the necessary national ethics, prison National Research Committee and local governance approvals, data was extracted retrospectively from the clinical records

of all new entrants to four remand (two male and two female) prisons between June 1 st and June $30^{\text {th }}, 2015$. All relevant data recorded in the clinical record within 12-months of arrival was extracted. Data extraction took place between June 2016 and June 2018. The rationale for retrospective data collection was that the research activity did not bias routine clinical practice which would have been a risk had data been collected prospectively. Data pertaining to the following were extracted: demographics (including age, gender, ethnic background and sentence status); length of stay in prison (categorised as less than or greater than six months); prevalence of the following "tracer" physical health LTCs: diabetes, asthma, hypertension, coronary heart disease, chronic obstructive pulmonary disease, epilepsy; prevalence of co-morbid mental health conditions; proportion of QOF templates completed - and whether completion was full or partial; time to completion of QOF template; supporting evidence for the long-term conditions defined as any one of: confirmation from the community GP, biochemical test or medication history. The above LTCs were selected as tracer conditions because they are the physical health LTCs that commonly present in prison first night receptions and because of their potential to cause significant morbidity and mortality. The LTCs were identified by the researchers through examining the patient's individual clinical record to retrieve self-reported information of the condition and whether it was confirmed by "supporting evidence." Supporting evidence was defined as any one of evidence of prescribed medication(s) indicated for the condition, 
confirmation from patient's community GP of the LTC or biomedical/clinical test confirming prevalence of the condition. The following biomedical/clinical tests were regarded as supporting evidence for prevalence:

- Diabetes - defined as an $\mathrm{HbA} 1 \mathrm{c}$ of $48 \mathrm{mmol} / \mathrm{mol}(6.5 \%)$ or above

- Asthma - (FEV1/FVC) ratio of less than $70 \%$ but positive reversibility test as diagnosed on spirometry (i.e. an increase in FEV 1 from baseline of $>12 \%$ in response to bronchodilators)

- Hypertension -blood pressure greater than $140 / 90 \mathrm{mmHg}$ or higher confirmed by either:

a) Ambulatory Blood Pressure Monitoring (ABPM) to confirm the diagnosis. ABPM confirmed a diagnosis through ensuring that at least two measurements per hour are taken during the person's usual waking hours, e.g. between 08:00 and 22:00 hours, and using the average value of at least 14 measurements taken during the person's usual waking hours to confirm a diagnosis of hypertension

OR

b) Home Blood Pressure Monitoring (HBPM) to confirm a diagnosis of hypertension through ensuring that for each blood pressure recording, two consecutive measurements are taken, at least 1 minute apart and with the person seated and blood pressure is recorded twice daily, ideally in the morning and evening and blood pressure recording continues for at least 4 days, ideally for 7 days. Discard the measurements taken on the first day and use the average value of all the remaining measurements to confirm a diagnosis of hypertension.

- Coronary Heart Disease - diagnosed by cardiologist from radiological findings

- COPD - spirometry highlighting airflow obstruction defined as FEV1 $<80 \%$ predicted and FEV1/FVC $<0.7$ which does not show reversibility to bronchodilator therapy

- Epilepsy - diagnosed by a neurologist (with or without supporting tests such as EEG or MRI)

Following data extraction, analysis was undertaken to assess:

- Prevalence of associated co-morbid physical and mental health conditions

- Proportion of prisoners still resident in the receiving study prison 6 months after entering and proportion with physical health or mental health conditions

- Proportion with a physical health LTC that had the relevant QOF template completed either partially or in full (prisoners residing in the prison for less than one-month were excluded to acknowledge the significant throughput of prisoners on short sentences in remand prisons which acts as a barrier to effective monitoring of LTCs).

- Time to completion of QOF template

- Qualification of the professional completing the QOF template

- Demographic associations with QOF completion

- Agreement between self-report in the primary care consultation of the LTC and confirmation with supporting evidence

Descriptive statistics are presented as mean (standard deviation (SD) or $\mathrm{n}(\%)$. Logistic regression analysis was undertaken of clinical data and the following statistical tests were undertaken: t-tests (continuous data), chi-square tests (categorical data) or Mann Whitney tests (ordinal data). Kappa was used to measure agreement between self-reported LTC and confirmation by supporting evidence. A $P$-value of $<0.05$ was considered to indicate statistical significance. Data analysis was undertaken in IBM SPSS Statistics for Windows (version 24). 
Results

In total, data was retrieved from the clinical records of 1,126 prisoners. Table 1 highlights the prisoner characteristics.

Of the prisoners, 185 (17\%) had at least one LTC. Regarding the association between total number of LTCs (i.e. diabetes, hypertension, asthma, CHD and COPD) and demographic characteristics, there was a significant difference by gender $(P$ $<0.001)$ and age $(P<0.001)$, but not ethnicity $(P=0.153)$. Females were more likely to have an LTC $(\mathrm{OR} 2.12 ; 95 \% \mathrm{Cl}$ : $1.50,3.00)$, and having an LTC was associated with older age (OR 1.05; 95\% Cl: 1.04, 1.07).

Table 2 highlights the number of prisoners with each of the LTCs in each of the prison sites as confirmed by supporting evidence (i.e. met the inclusion criteria for QOF monitoring). 221 LTCs were confirmed by supporting evidence, and the most common condition was asthma, which was confirmed in 12\% (135) of the sample. Epilepsy is excluded from this table since the epilepsy indicator was a "register only" (i.e. no clinical assessment required) in the 2015-16 QOF framework.

The prevalence rates of co-morbid mental health conditions in prisons were 31.5\% for depression, $24.9 \%$ for opioid dependence, $16 \%$ for alcohol dependence, $4.2 \%$ for schizophrenia, $19.9 \%$ for other psychotic illness and $26.5 \%$ for other neurotic illness. The prevalence rate for deep vein thrombosis (presented in this paper as a physical co-morbid condition associated with the co-morbid mental health condition of opioid dependence) was $1.7 \%$. Regarding residence in the receiving prison at six months, just 11\% (124) were still resident in the receiving study prison, whilst $75 \%(839)$ had been released and 14\% (155) had been transferred to another prison. Compared to those no longer in prison at six months, for those still in the receiving study prison, there was no significant difference in the prevalence of either a physical health LTC (OR 1.19, $P=0.487,95 \% \mathrm{Cl}: 0.73,1.93$ ) or co-morbid mental health condition (OR $1.35, P=0.112,95 \% \mathrm{Cl}: 0.93,1.97$ ).

Table 3 highlights QOF completion rates for each of the tracer conditions and shows low levels of QOF completion as evidenced in Table 4. Just 34\% (38/112) had a full QOF completed and 11 part-completed. There was significant variation for time to completion with a range of 5-358 days. 35 of the QOF templates were completed by a nurse and just one completed by a healthcare assistant ( 2 missing data). All of the 11 part-completed templates were undertaken by nursing professionals.

Table 5 highlights associations with QOF template completion (for those who had a QOF condition confirmed by supporting evidence) and prisoner characteristics. By univariate analysis, younger age $(P=0.028)$, male gender $(P<0.001)$ and prison site $(P<0.001)$ were statistically significantly associated with QOF completion. There was no significant difference by ethnicity $(P=0.956)$ or sentence status $(P=0.470)$. By multivariate analysis in a logistic regression model highlighted that only younger age $(P=0.015)$ and prison site $(P=0.017)$ remained statistically significant. 
The level of agreement between self-reported LTC and confirmation by supporting evidence was good for all conditions as highlighted in Table 6.

\section{Discussion}

\section{Summary}

Our findings highlight the significant challenges of managing LTCs in remand prison settings, as highlighted by the fact that $75 \%$ of prisoners were no longer in the receiving study prison six months after entering the prison. Of those still in prison, just $11 \%$ were still resident in the receiving study prison, whilst $14 \%$ had been transferred to another prison. The numbers were even higher for those with an LTC. Eighty-four percent of those with such a condition were no longer in the receiving study prison at six months. Regarding mental health, $54 \%$ of those with a co-morbid mental health condition were no longer in the receiving study prison at six months. Therefore, still being in the receiving study prison at six months was not associated with an increased likelihood of having either a co-morbid mental health or physical LTC.

Regarding prevalence, $17 \%$ of the sample had at least one LTC, the most common condition being asthma, confirmed in $12 \%$ of the sample. Having an LTC was associated with female gender and increasing age. The confirmed prevalence rates for the other LTCs were hypertension $3 \%$, coronary heart disease $2 \%$, diabetes $2 \%$ and chronic obstructive pulmonary disease $1 \%$. The prevalence of epilepsy was $3 \%$, but there was considerable variability between prisons. The likely reason for such variability is coding practice for patients with either pseudo seizures or alcohol withdrawal seizures. Our findings highlighted just $34 \%$ of the eligible sample had had a QOF template completed. QOF completion rates varied between LTCs and was highest for asthma, with a 40\% completion rate, and lowest for diabetes, with an $8 \%$ completion rate. Demographic variables were associated with QOF completion. Higher rates of completion were associated with younger age. There were also statistically significant inter-prison differences. This coupled with our finding of significant variation for time to completion triangulates with the findings in our linked paper reporting qualitative findings of differing clinical practice between prisons.

\section{Strengths and Limitations}

Our findings make a significant contribution to the evidence base regarding prevalence of LTCs in prison settings, which constitutes an under researched area. Further, in so far as we are aware, this research is the first study exploring existing processes regarding QOF monitoring in UK prisons. Whilst our study took place in four remand prisons, we are confident that our findings can be generalised across the remand prison estate, and also to training prisons, since all prisoners in such establishments have at some point been transferred from remand prisons.

Whilst extracting data from just four prisons could be perceived as a limitation, it remains the largest UK multi-centre research study to quantify LTC prevalence from clinical records and, at the only UK study exploring prison based QOF monitoring processes. 


\section{Comparison with Existing Literature}

Our prevalence statistics broadly concur with previous research conducted in this area which highlights respiratory disease as the most prevalent LTC in UK prison settings and prevalence statistics of lower than $5 \%$ for each of diabetes, heart disease or hypertension. ${ }^{[12]}$ Our research highlighted that the level of agreement between self-report in the primary care consultation upon first night reception and subsequent supporting evidence was good for all the LTCs. This concurs with a UK data-linkage study in which self-report survey findings regarding LTC prevalence were cross checked with prisoner primary care records and highlighted that level of agreement was good regarding the prevalence of physical LTCs. ${ }^{[3]}$

The community prevalence rates for LTCs in the UK has been recorded as the following; asthma $-8 \%$ ( $+3.6 \%$ compared to global prevalence); COPD - 1.8\% (-1.5\%); heart disease - 11.2\% (+2.2\%); diabetes - 7\% (-1.8\%), hypertension - 24.2\% (+9.2\%); epilepsy - 0.8\% (-0.1\%); opioid dependence - 0.4\% (equal to global prevalence); depression - $3 \%$ (-0.9\%); alcohol dependence $-0.9 \%(-0.5 \%)$ and schizophrenia $-0.95 \%$ (+0.67\%). Within the UK, 1 in 4 people will experience a mental health illness each year, the most common being General Anxiety Disorder (5.9\%) and depression (3\%).

Differences can, therefore, be ascertained between the prison population and the community population.

Implications for Research and/or Practice

The key implication for future research from our findings is that for future prevalence studies seeking to quantify morbidity of LTCs in prison settings, extraction and secondary data analysis of routinely collected clinical data will be as effective, but less costly than administering surveys to patients. We would recommend implementing research processes that fulfil the necessary requirements pertaining to robust information governance to facilitate extraction and anonymisation of routinely collected clinical data.

Regarding implications for practice, since LTC prevalence is associated with increasing age, yet our findings show an association between younger age and QOF completion, this presents a pressing training need to target QOF activity where the burden of disease is highest.

Such lack of engagement in QOF monitoring highlighted in our findings was despite a national requirement of prison providers through the Health and Justice Indicators of Performance (HJIPs) to report on QOF data. ${ }^{[12]}$ The relevant document states: "Through the use of SystmOne templates and standard reporting, providers are able to self-assess their LTC monitoring, and report this as part of their HJIP data submission; providing performance outcomes against the chronic disease register and achievement against nationally recognised quality outcomes framework. This reporting enables assurance that there is parity of treatment provision between residents of the secure estate and the wider community. Providers are able to access their QOF achievement outcomes via a report embedded in SystmOne." Therefore, there appears to be a disconnect between national reporting requirements and clinical activity "on the ground." This could be addressed, in part, through the pending developments in the electronic patient record linkage systems, whereby community and prison GP electronic clinical records will be better linked. 
In our linked paper reporting qualitative findings, such a prospect was universally welcomed by participants and, in addition to better meeting acute health need, it was felt that such a development would support seamless monitoring of QOF activity between community and prison. Therefore, this presents an opportunity to introduce QOF monitoring systems, possibly supported by an integrated community/prison commissioning framework to enable future connectivity between prison and community primary care records of not just the "summary care record" (a minimum dataset comprising: current medication; allergies and details of any previous bad reactions to medicines; name, address, date of birth and NHS number of the patient) ${ }^{[13]}$ but also all activity related to QOF compliance.

Page BreakTables

\section{Table 1: Demographic characteristics}

\begin{tabular}{|c|c|c|}
\hline & & Mean (sd), min-max OR n (\%) \\
\hline \multicolumn{2}{|l|}{ Age } & $35.0(10.4) 19-80$ \\
\hline \multirow{3}{*}{ Gender } & Male & $882(78 \%)$ \\
\hline & Female & $243(22 \%)$ \\
\hline & Undetermined & $1(<1 \%)$ \\
\hline \multirow{6}{*}{ Ethnicity } & Asian or Asian British & $47(4 \%)$ \\
\hline & Black or Black British & $19(2 \%)$ \\
\hline & Mixed & $47(4 \%)$ \\
\hline & White & $691(61 \%)$ \\
\hline & Other & $154(14 \%)$ \\
\hline & Missing & $168(15 \%)$ \\
\hline \multirow{6}{*}{ Sentence status } & Remand & $381(34 \%)$ \\
\hline & License revoke & $68(6 \%)$ \\
\hline & Trial & $1(<1 \%)$ \\
\hline & Sentenced & $520(46 \%)$ \\
\hline & Unknown & $152(14 \%)$ \\
\hline & Missing & $4(<1 \%)$ \\
\hline \multirow{4}{*}{ Prison } & Prison A & $355(31 \%)$ \\
\hline & Prison B & $76(7 \%)$ \\
\hline & Prison C & $167(15 \%)$ \\
\hline & Prison D & $528(47 \%)$ \\
\hline
\end{tabular}

Table 2: Proportion of LTCs in each prison site 


\begin{tabular}{|c|c|c|c|c|c|c|c|c|c|c|}
\hline & & \multicolumn{2}{|c|}{ Prison A } & \multicolumn{2}{|c|}{ Prison B } & \multicolumn{2}{|c|}{ Prison C } & \multicolumn{2}{|c|}{ Prison D } & \multirow[t]{2}{*}{ Total } \\
\hline & & $\mathrm{n}$ & $\%$ & $\mathrm{n}$ & $\%$ & $\mathrm{n}$ & $\%$ & $\mathrm{n}$ & $\%$ & \\
\hline \multirow{2}{*}{ Diabetes confirmed by supporting evidence? } & Yes & 7 & $30.4 \%$ & 2 & $8.7 \%$ & 5 & $21.7 \%$ & 9 & $39.1 \%$ & 23 \\
\hline & No & 348 & $31.6 \%$ & 74 & $6.7 \%$ & 162 & $14.7 \%$ & 519 & $47.1 \%$ & 1103 \\
\hline \multirow{2}{*}{ Asthma confirmed by supporting evidence? } & Yes & 47 & $34.8 \%$ & 11 & $8.1 \%$ & 36 & $26.7 \%$ & 41 & $30.4 \%$ & 135 \\
\hline & No & 308 & $31.1 \%$ & 65 & $6.6 \%$ & 131 & $13.2 \%$ & 487 & $49.1 \%$ & 991 \\
\hline \multirow{2}{*}{ Hypertension confirmed by supporting evidence? } & Yes & 11 & $31.4 \%$ & 2 & $5.7 \%$ & 10 & $28.6 \%$ & 12 & $34.3 \%$ & 35 \\
\hline & No & 344 & $31.5 \%$ & 74 & $6.8 \%$ & 157 & $14.4 \%$ & 516 & $47.3 \%$ & 1091 \\
\hline \multirow{2}{*}{ CHD confirmed by supporting evidence? } & Yes & 6 & $35.3 \%$ & 0 & $0.0 \%$ & 2 & $11.8 \%$ & 9 & $52.9 \%$ & 17 \\
\hline & No & 349 & $31.5 \%$ & 76 & $6.9 \%$ & 165 & $14.9 \%$ & 519 & $46.8 \%$ & 1109 \\
\hline \multirow{2}{*}{ COPD confirmed by supporting evidence? } & Yes & 1 & $9.1 \%$ & 1 & $9.1 \%$ & 7 & $63.6 \%$ & 2 & $18.2 \%$ & 11 \\
\hline & No & 354 & $31.7 \%$ & 75 & $6.7 \%$ & 160 & $14.3 \%$ & 526 & $47.2 \%$ & 1115 \\
\hline
\end{tabular}

\section{Table 3 - QOF completion rates for each tracer condition}

\begin{tabular}{ll|l|l|l} 
& \multicolumn{4}{l}{ Diabetes confirmed by supporting evidence? } \\
\hline & Yes & No & \\
\hline QOF Completion for Diabetes & N & $\%$ & n & $\%$ \\
\hline Yes & 1 & $4.3 \%$ & 0 & $0 \%$ \\
\hline No & 12 & $52.2 \%$ & 1 & $<1 \%$ \\
\hline Not applicable & 3 & $13 \%$ & 1101 & $99 \%$ \\
\hline No as in prison for less than 1 month & 4 & $17.4 \%$ & 1 & $<1 \%$ \\
\hline No because not due & 1 & $4.3 \%$ & 0 & $0 \%$ \\
\hline No as patient DNAed & 1 & $4.3 \%$ & 0 & $0 \%$ \\
\hline Part done & 1 & $4.3 \%$ & 0 & $0 \%$ \\
\hline Total & 23 & $100.0 \%$ & 1103 & $100.0 \%$ \\
\hline
\end{tabular}

\section{Asthma confirmed by supporting evidence?}

QOF completion for asthma
Yes
No
Not applicable
No as in less than 1 month
No because not due
No as patient DNAed
Part done
Total

Total

QOF completion for hypertension

Yes

\begin{tabular}{|l|l|l|l|}
\hline Yes & \multicolumn{3}{|l}{ No } \\
\hline $\mathbf{n}$ & $\%$ & $\mathbf{n}$ & $\%$ \\
\hline 32 & $23.7 \%$ & 1 & $<1 \%$ \\
\hline 48 & $35.6 \%$ & 34 & $3.4 \%$ \\
\hline 5 & $3.7 \%$ & 950 & $95.9 \%$ \\
\hline 34 & $25.2 \%$ & 2 & $<1 \%$ \\
\hline 4 & $3 \%$ & 0 & $0 \%$ \\
\hline 3 & $2.2 \%$ & 1 & $<1 \%$ \\
\hline 9 & $6.7 \%$ & 2 & $<1 \%$ \\
\hline 135 & $100 \%$ & 991 & $100.0 \%$ \\
\hline
\end{tabular}

Hypertension confirmed by supporting evidence?

Yes No

\begin{tabular}{|l|l|l|l|}
\hline $\mathbf{n}$ & $\%$ & $\mathbf{n}$ & $\%$ \\
\hline 4 & $11.4 \%$ & 0 & $0 \%$ \\
\hline
\end{tabular}




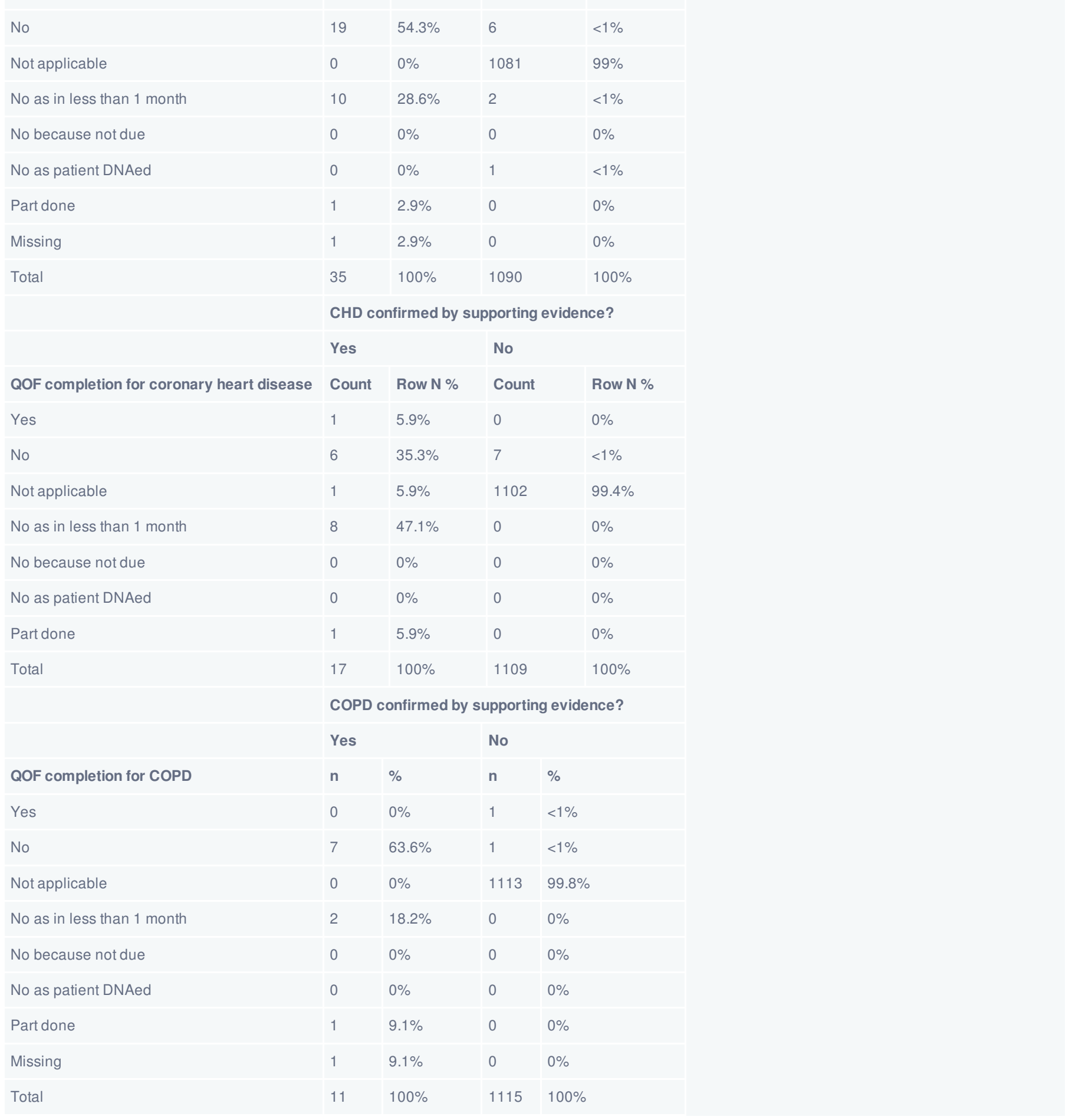

Table 4 - QOF condition confirmed by higher evidence and QOF completion 


\begin{tabular}{|l|l|l|l|l|l|l|}
\hline & & \multicolumn{2}{|c|}{ Number of LTCs } & & Total \\
\hline & & $\mathbf{0}$ & $\mathbf{1}$ & $\mathbf{2}$ & $\mathbf{3}$ & \\
\hline & Yes & 0 & 36 & 2 & 0 & 38 \\
\hline & No & 0 & 61 & 10 & 3 & 74 \\
\hline & Not applicable & 941 & 0 & 0 & 0 & 947 \\
\hline $\begin{array}{l}\text { QOF } \\
\text { (number of patients) }\end{array}$ & No as in less than 1 month & 0 & 37 & 6 & 4 & 47 \\
\hline & No because not due & 0 & 10 & 0 & 1 & 5 \\
\hline & No as patient DNAed & 0 & 4 & 0 & 0 & 4 \\
\hline & Part done & 0 & 9 & 2 & 0 & 11 \\
\hline & & 941 & 157 & 20 & 8 & 1126 \\
\hline
\end{tabular}

Table 5: Characteristics of those who did and did not have QOF completed

\begin{tabular}{|c|c|c|c|c|c|}
\hline & & \multicolumn{2}{|c|}{ Yes QOF $(n=38)$} & No QOF $(n=74)$ & \\
\hline & & $\mathrm{n}$ & $\%$ & $\%$ & \\
\hline Age (Mean (SD)) & & \multicolumn{2}{|c|}{37.3 (SD: 12.2) } & 42.8 (SD: 12.3) & \\
\hline \multirow{4}{*}{ Prison site } & Prison A & 29 & $78 \%$ & $22 \%$ & \\
\hline & Prison B & 3 & $27 \%$ & $73 \%$ & \\
\hline & Prison C & 0 & $0 \%$ & $100 \%$ & \\
\hline & Prison D & 6 & $18 \%$ & $82 \%$ & \\
\hline \multirow{2}{*}{ Gender } & Male & 35 & $50 \%$ & $50 \%$ & \\
\hline & Female & 3 & $7 \%$ & $93 \%$ & \\
\hline \multirow{6}{*}{ Ethnicity } & Asian or Asian British & 1 & $33 \%$ & $67 \%$ & \\
\hline & Black or Black British & 0 & $0 \%$ & $100 \%$ & \\
\hline & Mixed & 1 & $20 \%$ & $80 \%$ & \\
\hline & White & 18 & $24 \%$ & $76 \%$ & \\
\hline & Other & 1 & $17 \%$ & $83 \%$ & \\
\hline & Missing & 17 & $81 \%$ & $19 \%$ & \\
\hline \multirow{5}{*}{ Sentence status } & Remand & 12 & $33 \%$ & $67 \%$ & \\
\hline & License revoke & 0 & $0 \%$ & $100 \%$ & \\
\hline & Trial & 0 & $0 \%$ & $0 \%$ & \\
\hline & Sentenced & 21 & $32 \%$ & $68 \%$ & \\
\hline & Unknown & 5 & $56 \%$ & $44 \%$ & \\
\hline
\end{tabular}

Table 6: Level of agreement between self-report and confirmed by supporting evidence 


\begin{tabular}{|c|c|c|c|c|c|}
\hline & & \multicolumn{2}{|c|}{ Confirmed by supporting evidence? } & \multirow{2}{*}{ Total } & \multirow{2}{*}{ Kappa, p-value } \\
\hline & & Yes & No & & \\
\hline \multirow{2}{*}{ Diabetes self-reported? } & Yes & 15 & 2 & 17 & \multirow{2}{*}{$0.746, p<0.001$} \\
\hline & No & 8 & 1101 & 1109 & \\
\hline \multirow{2}{*}{ Asthma self-reported? } & Yes & 119 & 38 & 157 & \multirow{2}{*}{$0.791, p<0.001$} \\
\hline & No & 15 & 953 & 968 & \\
\hline \multirow[t]{2}{*}{ Hypertension self-reported? } & Yes & 27 & 7 & 34 & \multirow[t]{2}{*}{$0.776, p<0.001$} \\
\hline & No & 8 & 1080 & 1088 & \\
\hline \multirow{2}{*}{ CHD self-reported? } & Yes & 12 & 6 & 18 & \multirow{2}{*}{$0.681, p<0.001$} \\
\hline & No & 5 & 1103 & 1108 & \\
\hline COPD self-reported? & Yes & 9 & 3 & 12 & $0.780, p<0.001$ \\
\hline
\end{tabular}

Page Break

\section{References}

1. ${ }^{a, b}$ Department of Health. (2013). Long Term Conditions Compendium of Information: Third Edition.. Department of Health.

2. ^Angela Coulter et al. (2013). Delivering better services for people with long-term conditions. Building the house of care.. King's Fund.

3. ${ }^{a}{ }^{b}$ Nat MJ Wright, Philippa Hearty, Victoria Allgar. (2019). Prison primary care and non-communicable diseases: a data-linkage survey of prevalence and associated risk factors. BJGP Open, vol. 3 (2), bjgpopen19X101643. doi:10.3399/bjgpopen19×101643.

4. ^House of Commons Library. (2018). UK Prison Population Statistics: Briefing Paper Number CBP-04334. UK Parliament.

5. 'T Marshall. (2001). Use of health services by prison inmates: comparisons with the community. doi:10.1136/jech.55.5.364.

6. 'Louise Condon, Hek Gill, Francesca Harris. (2007). A review of prison health and its implications for primary care nursing in England and Wales: the research evidence. J Clin Nurs, vol. 16 (7), 1201-1209. doi:10.1111/j.13652702.2007.01799.x.

7. ^Royal College of General Practitioners. (2018). RCGP statement on care in secure environments. RCGP.

8. ^Medical Defence Union. (2010). Treatment Behind Bars. MDU Journal, vol. 26 (1). MDU.

9. ^National Prescribing Centre. (2002). Medicines management services - why are they important? In: NICE - Medicines optimisation: the safe and effective use of medicines to enable the best possible outcomes. National Prescribing Centre. 
10. ^British Medical Association.. (2019). The Quality and Outcomes Framework (QOF). BMA.

11. ^ISD Scotland.. (2019). Primary Care Information. Public Health Scotland.

12. ${ }^{a,}$ bNS England. (2017). Health and Justice Indicators of Performance (HJIPs): Adult Secure Estate User Guide 201718. V1.5.. NHSE.

13. 'NHS Digital.. (2019). Quality and Outcomes Framework, Achievement, prevalence and exceptions data - 2017-18. NHS Digital. 